\title{
Motor pathway function in normoalbuminuric IDDM patients
}

\author{
H. Andersen ${ }^{1}$, J. F. Nielsen ${ }^{1}$, P. L. Poulsen ${ }^{2}$, C.E. Mogensen ${ }^{2}$, J. Jakobsen ${ }^{1}$ \\ ${ }^{1}$ Department of Neurology, Aarhus University Hospital, Aarhus, Denmark \\ ${ }^{2}$ Second University Clinic of Internal Medicine, Aarhus University Hospital, Aarhus, Denmark
}

Summary Central motor pathways were studied in 17 normoalbuminuric insulin-dependent diabetic (IDDM) patients who had been diabetic for more than 20 years, and compared with findings in 17 age-, sex-, and height-matched control subjects. The central motor conduction time was calculated from recordings of the compound muscle action potentials of the abductor pollicis brevis muscle after single transcranial and spinal root magnetic stimulation. The central motor conduction time from motor cortex to cervical spinal roots was $9.8 \pm 1.65 \mathrm{~ms}$ in diabetic patients and $10.1 \pm 1.48 \mathrm{~ms}$ in control subjects. In diabetic patients with neuropathy the central motor conduction time was $9.5 \pm 1.76 \mathrm{~ms}$ vs $10.1 \pm 1.56$ $\mathrm{ms}$ in patients without neuropathy. The excitability of the motor pathways was studied by paired transcranial magnetic stimulation at interstimulation intervals of 30-1000 ms. In normal control subjects, an early facilitation of the amplitude of the compound muscle action potential at an interstimulation interval of $30 \mathrm{~ms}$ was found, while no facilitation was present in diabetic patients. In addition the compound muscle action potential latencies were prolonged at interstimulation intervals of $30-50 \mathrm{~ms}$ in diabetic patients. The changes of excitability did not correlate with the presence of peripheral neuropathy, metabolic control or diabetes duration. It is concluded that long-term normoalbuminuric IDDM patients have impaired excitability but normal central conduction time of the motor pathways. [Diabetologia (1995) 38: 1191-1196]

Key words Magnetic stimulation, diabetic neuropathy, motor pathway, paired magnetic stimulation, diabetic encephalopathy.
Motor, sensory and autonomic disturbances are concomitants with diabetic neuropathy. Sensory and autonomic dysfunctions have been well-studied and a battery of cutaneous perception threshold tests and autonomic function tests to characterize and monitor the neuropathy have been developed and validated.

Received: 31 October 1994 and in revised form: 15 March 1995

Corresponding author: Dr. H. Andersen, Department of Neurology, Aarhus University Hospital, Nørrebrogade 44, DK8000 Aarhus C, Denmark

Abbreviations: CMCT, Central motor conduction time; CMAP, compound muscle action potential; ISI, interstimulation interval, $\mathrm{MNCV}$, motor nerve conduction velocity; SNCV, sensory nerve conduction velocity; APB, abductor pollicis brevis muscle; IDDM, insulin-dependent diabetes mellitus; NIDDM, non-insulin-dependent diabetes mellitus.
In contrast, less is known about motor function in diabetic neuropathy. It has been well-described that peripheral motor nerve function is electrophysiologically impaired to the same degree as sensory nerve function, but apart from this the motor system from the motor cortex to striated muscle has been studied very little in diabetes. An explanation could be the predominance of sensory symptoms and signs in diabetic neuropathy. A recent population-based epidemiological study of diabetic neuropathy demonstrated, however, that clinical motor weakness is a severe disturbance in patients with advanced neuropathy [1]. This observation clearly indicates the need for further studies of motor function in diabetic patients.

With the development of the magnetic stimulation technique it has become possible with single transcra- 
nial stimuli of the motor cortex and the nerve roots to assess central conduction time (CMCT) of the motor pathways [2]. Recently, in a mixed group of insulindependent (IDDM) and non-insulin-dependent diabetic (NIDDM) patients Abbruzzese et al. [3] studied CMCT with single transcranial magnetic stimulation. They found that $30 \%$ of the diabetic patients had prolonged CMCT from the cortex to cervical roots. The prolongation of the conduction time was unrelated to the presence of peripheral neuropathy indicating independent dysfunction of central motor pathways. In addition transcranial magnetic stimulation allows evaluation of the excitability of the motor pathways by paired stimuli of the motor cortex. With this new technique the time dependency of excitability is studied by varying the interstimulation interval (ISI) between the conditioning stimulus and the test stimulus $[4,5]$.

CMCT and excitability of the motor pathways from motor cortex to striated muscle were studied in normoalbuminuric IDDM patients with single and paired transcranial magnetic stimulation. To secure a high prevalence of neuropathy in the diabetic group patients with more than 20 years of diabetes duration were studied. Only patients with normoalbuminuria were included in order to avoid the influence of any nephropathic complication on the motor pathways.

\section{Subjects and methods}

Patients and control subjects. Nineteen normoalbuminuric IDDM patients from the out-patient clinic, 6 females and 13 males, aged 43 years (range: 31-58) with a height of $174 \mathrm{~cm}$ (range: 165-185) and a median diabetes duration of 28 years (range: 20-37) were included in the study. Seventeen sex-, age-, and height-matched control subjects had a mean age of 44 years (range: $31-60$ ), and a height of $174 \mathrm{~cm}$ (range: $163-$ 194). None of the patients or control subjects had a history of any central nervous system disorder. The patients gave informed consent to the study and the protocol was approved by the local ethical committee.

Clinical and electrophysiological evaluation. All patients were examined by a trained neurologist and evaluated with a neuropathy symptom score and a neurological disability score [6, 7]. $\mathrm{HbA}_{1}$, blood glucose and serum creatinine were measured at the time of the study. Urinary albumin excretion was determined by radioimmunoassay and normoalbuminuria was defined as an albumin excretion rate of less than $20 \mu \mathrm{g} / \mathrm{min}$ [8]. Nerve conduction studies were performed with standard surface stimulation and recording techniques using an electromyograph (DANTEC Counterpoint, Skovlunde, Denmark) with filter settings of $20 \mathrm{~Hz}$ to $10 \mathrm{kHz}$ for motor nerve studies and $20 \mathrm{~Hz}$ to $2 \mathrm{kHz}$ for sensory nerve studies.

Motor nerve conduction velocity (MNCV) was measured in the dominant forearm segment of the median (elbow wrist) nerve and in the non-dominant leg segment of the peroneal (below capitulum fibulae - ankle) nerve. Sensory nerve conduction velocity (SNCV) was measured in the non-dominant sural nerve with orthodromic activation and with antidro- mic activation of the dominant median nerve (wrist - finger II and III). For MNCV Z-scores were calculated from values of healthy volunteers obtained with similar techniques [9]. For SNCV normal values previously determined in age-matched normal control subjects were adopted. Cutaneous sensation was evaluated quantitatively by measuring vibratory perception threshold at dominant index fingerpulp and non-dominant dorsum of the great toe using forced choice techniques using a CASE IV (WR Medical Electronics Co., Stillwater, Minn., USA). Prior to the examination patients were given written instructions and a demonstration of the technique. The threshold was determined with the 4,2, and 1 stepping algorithm [10]. The minimal criteria for diabetic neuropathy were adopted in a slightly modified form [11, 12]. Patients with at least two abnormal findings of the following four were defined as neuropathic: (a) neuropathy symptom score $\geq 1$; (b) neurological disability score $\geq 2$; (c) abnormal nerve conduction velocity in at least two out of four nerves; (d) abnormal vibratory perception threshold at both sites.

Magnetic stimulation and recording. Compound muscle action potentials (CMAPs) were recorded from the abductor pollicis brevis (APB) on the dominant side with $\mathrm{Ag} / \mathrm{AgCl}$ electrodes placed in a belly-tendon montage. To ensure absolute muscle relaxation during stimulation a modified myometer gave an on-line audio/visual-monitoring of muscle activity at maximum gain with subjects awake in a comfortable supine position [13]. During transcranial stimulation the coil was centered at the vertex. The CMAPs were recorded with the DANTEC Counterpoint with filter settings of $20 \mathrm{~Hz}$ to $2 \mathrm{kHz}$. To avoid any post-stimulation effect the interval between stimuli was at least $5 \mathrm{~s}$.

Single and paired magnetic cortical pulses were given by a magnetic stimulator constructed in our laboratory. It renders a biphasic pulse with a current rise time of $200 \mu \mathrm{s}$ in a $13-\mathrm{cm}$ diameter coil with a maximal magnetic field of 2.2 Tesla. The output was adjustable between 0 and 1200 V. For determination of the threshold stimulation intensity was increased by $50-\mathrm{V}$ steps and defined as the intensity at which three of four stimuli evoked well-defined CMAPs at a gain of $100 \mu \mathrm{V}$. For paired stimuli the intensity of the test and the conditioning stimulus were constant at all ISIs being 110 and $152 \%$ of threshold intensity, respectively.

The following magnetic studies were conducted: (A) CMCT was calculated by subtraction of the spinal latency (C7 to APB) from the cortical latency (cortex to APB). The cortical latency was determined by single transcranial magnetic stimuli at $120 \%$ of threshold intensity. In order to excite the spinal nerves close to the exit foramina the magnetic coil was positioned over the spinous process of C-7 and the edge of the coil $3 \mathrm{~cm}$ lateral to the process. The cortical and spinal latency were determined as the shortest latency of the four CMAPs. (B) Four paired magnetic stimuli were randomly given at ISIs of $30,40,50,75,100,150,200,250,300,500$, and $1000 \mathrm{~ms}$ and recorded from the APB at rest. As control values four single stimuli were given at a stimulation intensity of $110 \%$ of threshold.

\section{Statistical analysis}

For data analysis, take-off latency and baseline-to-peak amplitude were determined at maximum gain. In case no response was obtained following the conditioned stimulus a zero value was included for the arithmetic median calculation of amplitudes. 
Table 1. Take-off latencies, CMCT and baseline-to-peak amplitudes of single cortical and spinal evoked CMAPs of the abductor pollicis brevis muscle in diabetic patients and normal control subjects

\begin{tabular}{lcl}
\hline & $\begin{array}{l}\text { IDDM patients } \\
(n=17)\end{array}$ & $\begin{array}{l}\text { Control subjects } \\
(n=17)\end{array}$ \\
\hline $\begin{array}{l}\text { Latency }(m s) \\
\text { cortical }\end{array}$ & $24.1 \pm 2.06$ & $23.3 \pm 1.42$ \\
spinal & $14.3 \pm 1.90$ & $13.2 \pm 1.83$ \\
CMCT $(m s)$ & $9.8 \pm 1.65$ & $10.1 \pm 1.48$ \\
Amplitude $(\mu V)$ & & \\
cortical & $164(77.270)$ & $226(170.322)$ \\
spinal & $199(109.415)$ & $338(220.840)$ \\
\hline
\end{tabular}

Data given as mean \pm SD for latencies and CMCT and median and $25^{\text {th }}, 75^{\text {th }}$ percentiles for baseline-to-peak amplitudes

For statistical analysis Systat (Version 5.0, Systat, Evanston, Ill., USA) was applied. Latency data were analysed with twotailed Students $t$-test for paired and unpaired data, whereas two-sided Mann-Whitney and Wilcoxon signed rank tests were employed for amplitude data using a $5 \%$ limit of statistical significance.

\section{Results}

In patients $\mathrm{HbA}_{1 \mathrm{c}}$ was $9.0 \%$ (range $7.2-10.9$ ), serum creatinine $81.5 \mathrm{umol} / \mathrm{l}$ (range 61-108) and blood glucose level $10.5 \mathrm{mmol} / 1$ (range 2.1-19.9). The neuropathy symptom score assessment revealed 13 asymptomatic and 4 symptomatic patients. The neurological disability score was 6 (range $0-36$ ). MNCV and the corresponding Z-score for the median and peroneal nerves were $53.4 \mathrm{~m} / \mathrm{s}$ (range 41.0-56.6), 1.13 (range 0.11-4.39) and $40.4 \mathrm{~m} / \mathrm{s}$ (range 37.4-48.9), 2.03 (range 0.07-3.01), respectively. SNCV for the median and sural nerve were $48.2 \mathrm{~m} / \mathrm{s}$ (range $39.7-$ 48.9 ) and $46.3 \mathrm{~m} / \mathrm{s}$ (range $40.5-55.8$ ), respectively. In three patients no action potentials of the median and sural nerves could be detected, one patient refused further examination after median nerve examination and in one patient conduction velocity of the peroneal nerve could not be determined. According to the minimal criteria for diabetic neuropathy nine patients were classified as "neuropathic" and eight patients as "non-neuropathic".

Magnetic stimulation studies. Two patients were excluded from all magnetic stimulation studies because threshold values exceeded $1000 \mathrm{~V}$ and one patient was excluded from paired magnetic stimulation studies because the threshold exceeded $875 \mathrm{~V}$.

No difference in the CMCT following single magnetic stimulation in diabetic patients and control subjects was found (Table 1). Neither was there any difference between the neuropathic $(9.5 \pm 1.76 \mathrm{~ms})$ $(n=9)$ and non-neuropathic $(10.1 \pm 1.56 \mathrm{~ms})$ patients $(n=8)$. The amplitude following single corti- cal and spinal stimulation was unchanged in diabetic patients as compared to control subjects (Table 1). The amplitude following single cortical stimulation was unchanged in neuropathic as compared to nonneuropathic patients, $266 \mu \mathrm{V}$ (range 18-2554) and $131 \mu \mathrm{V}$ (range 28-1285), respectively. Following single spinal stimulation the amplitude was unchanged in neuropathic as compared to non-neuropathic patients, $199 \mu \mathrm{V}$ (range 37-2006) and $214 \mu \mathrm{V}$ (range $84-1275)$, respectively. There was no correlation between CMCT and either $\mathrm{HbA}_{1 \mathrm{c}}(r=-0.51, p>0.07)$, MNCV of the median nerve $(r=-0.07, p>0.83)$ or duration of diabetes $(r=-0.27, p>0.38)$.

Paired magnetic stimulation resulted in a facilitation of the amplitude of the CMAPs at an ISI of 30 ms in control subjects and subsequently a depression was seen for the ISIs of 75-200 ms with no CMAPs evoked in any subject at the interval of $75-150 \mathrm{~ms}$. In contrast, in diabetic patients no facilitation of the response at ISI of $30 \mathrm{~ms}$ occurred and a depression of the amplitude was present for the ISI interval 50-250 ms (Fig. 1). No correlation was found between amplitude and either MNCV, HbA1 c or duration of diabetes $(r=0.43, p>0.14 ; r=-0.32, p>0.28 ; r=-0.51$, $p>0.07$, respectively). During paired stimulation latency of the CMAP showed a significant increase in the diabetic group at ISIs of $30-50 \mathrm{~ms}$ and also at the ISI of $500 \mathrm{~ms}$, whereas no changes in latency were found in control subjects (Fig. 2). There was no correlation between latency at the ISI of $30 \mathrm{~ms}$ and either $\mathrm{MNCV}, \mathrm{HbA}_{1 \mathrm{c}}$, or duration of diabetes $(r=-0.25$, $p>0.40 ; r=0.34, p>0.26 ; r=0.17, p>0.58$, respectively).

\section{Discussion}

The major findings in this study are lack of facilitation of the CMAP at ISI of $30 \mathrm{~ms}$ as well as increased latency at ISIs of $30-50 \mathrm{~ms}$ following paired magnetic stimulation in long-term normoalbuminuric IDDM patients. In contrast the CMCT was unchanged in diabetic patients and the CMCTs in neuropathic and non-neuropathic patients were similar.

Manifestations of impaired cerebral function in diabetic patients have been demonstrated with other electrophysiological methods. Increased latencies of visual, auditory, and somatosensory evoked potentials have been reported in diabetic patients [14-16]. An increase of the P300 wave latency has also been demonstrated which may reflect impairment of higher brain functions [17]. In addition neurochemical, structural, and neurobehavioural manifestations of cerebral dysfunction in diabetes have been reported in both experimental and human diabetes [18].

With the introduction of the non-invasive magnetic stimulation technique it has become possible to study central motor pathways [2]. There are two re- 

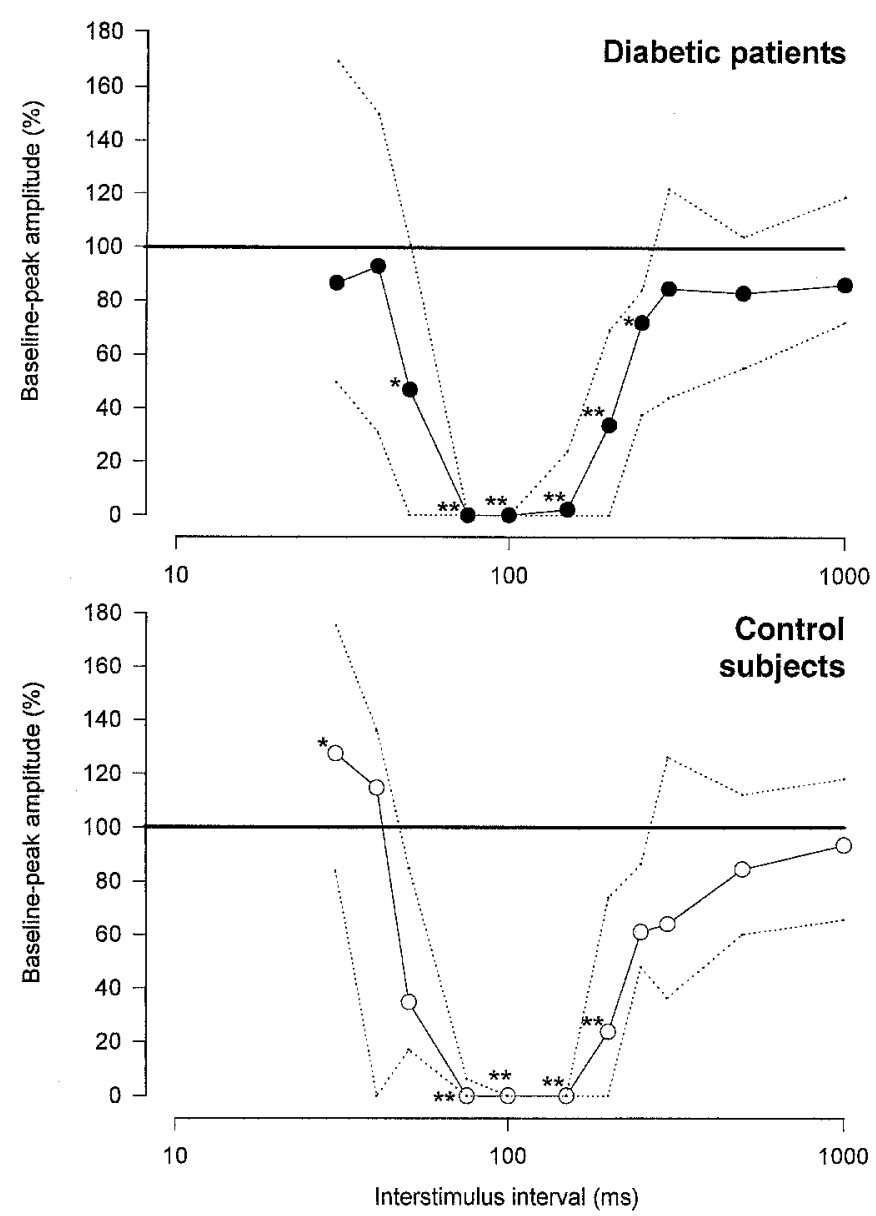

Fig. 1. Median values ( - ) and the 25 th and 75 th percentiles $(\cdots)$ of baseline-peak amplitudes of CMAPs of abductor pollicis brevis muscle evoked by a magnetic stimulus at a stimulation intensity of $110 \%$ of threshold intensity conditioned by a suprathreshold magnetic stimulus. $* p<0.05, * * p<0.01$

cent studies of the central motor pathways in diabetic patients $[3,19]$. In contrast to the present study both these studies reported a prolonged CMCT in diabetic patients. In a mixed diabetic population, $\mathrm{Ab}-$ bruzzese et al. [3] reported a prolongation of the $\mathrm{CMCT}$, which closely correlated with metabolic control (high $\mathrm{HbA}_{1 \mathrm{c}}$ ) without any correlation with peripheral neuropathy or microangiopathy. Tchen et al. [19] also found a prolonged CMCT which in their study correlated with the duration of diabetes and impairment of the peripheral nervous system. In the present study no correlation between CMCT and either $\mathrm{HbA}_{1 c}$, duration of diabetes or peripheral neuropathy was found. These conflicting results may be ascribed to heterogeneity in type and duration of diabetes, and to a different frequency of renal complications in the study populations. The diabetic population in the studies by Abbruzzese et al. [3] and Tchen et al. [19] differed in several ways from the diabetic population in this study. The major differences were that about two-thirds of the patients had NIDDM, the mean age was higher and in the study by $\mathrm{Ab}$ -
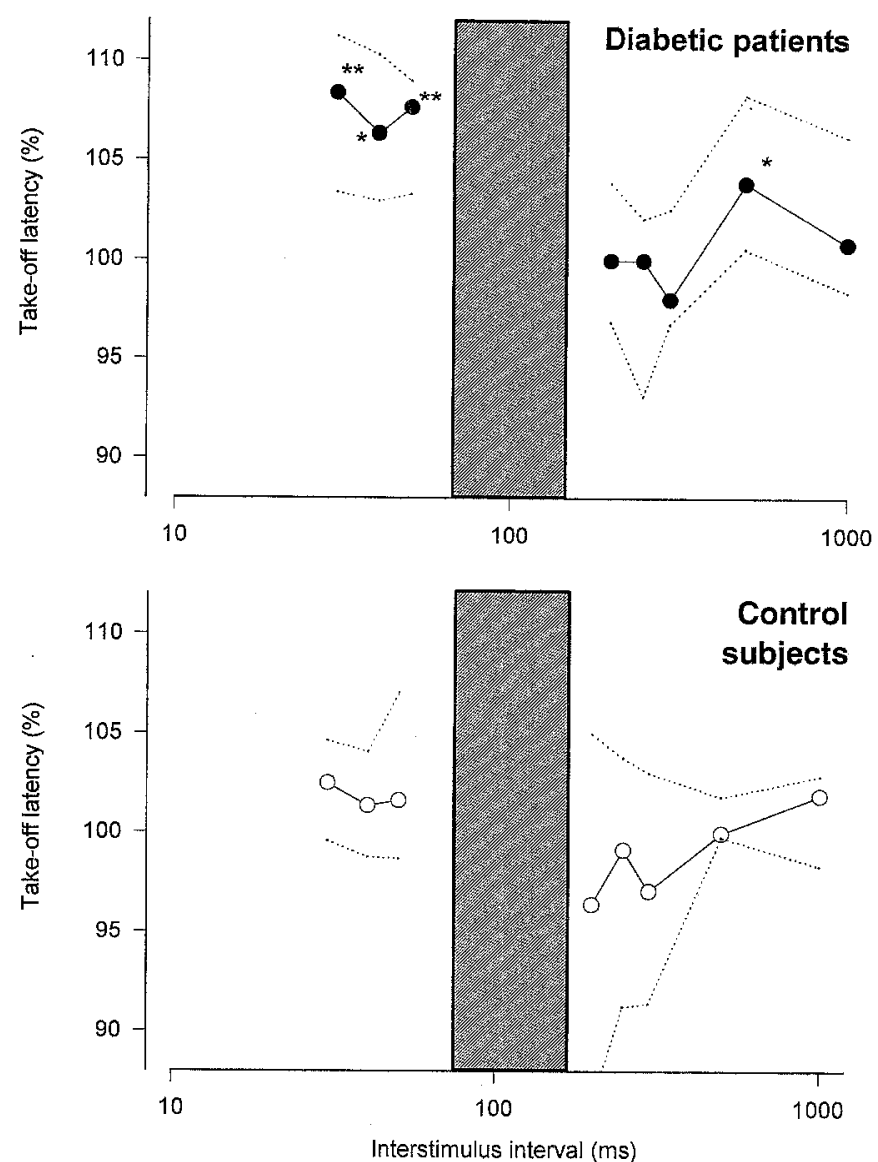

Fig. 2. Median values (-) and the 25 th and 75 th percentiles $(\cdots)$ of take-off latencies of CMAPs of abductor pollicis brevis muscle evoked by a magnetic stimulus at a stimulation intensity of $110 \%$ of threshold intensity conditioned by a suprathreshold magnetic stimulus. The bar indicates the interval when no CMAP was obtained. * $p<0.05,{ }^{* *} p<0.01$

bruzzese et al. more than $15 \%$ had signs of nephropathy while Tchen et al. did not report the prevalence of renal complications in the diabetic group. In contrast all the patients in this study had IDDM and all were normoalbuminuric.

In a study by Pietravalle et al. [20] subclinical kidney abnormalities in long-term IDDM patients correlated with CNS abnormalities evaluated by multimodal evoked potentials. Although they also found CNS abnormalities in $27 \%$ of short-term diabetic patients without kidney abnormalities the authors suggested the sharing of some pathogenetic factors between kidney and CNS abnormalities. Irrespective of these pathogenetic mechanisms being unknown, the finding of a normal CMCT in this study may partly be explained by the absence of any kidney abnormality in the diabetic group.

Normal CMCT in the diabetic group suggests a normal function of the motor pathways. However, applying paired magnetic stimulation, the lack of facilitation and the increased latency of the CMAPs in diabetic patients indicate an altered excitability. A facili- 
tation of the response at ISI of $30 \mathrm{~ms}$ would be expected in accordance with findings in the control group and in the literature. Valls-Solé et al. [4] applied paired transcranial magnetic stimuli at high intensity (120-150\% of threshold intensity) and found facilitation of CMAPs from the APB at ISIs of 25-50 $\mathrm{ms}$ followed by inhibition at ISIs of $60-150 \mathrm{~ms}$, and occasionally by a secondary phase of facilitation at ISIs of more than $200 \mathrm{~ms}$. Similar results have been obtained in a study of paired magnetic stimulation in our laboratory. We observed a facilitation of amplitudes of CMAPs at ISIs of 15-50 ms obtained from the first dorsal interosseous muscle and from m. flexor carpi radialis followed by an inhibition of the response to test stimuli with maximum at an of ISI of $100 \mathrm{~ms}$ where no responses could be evoked (Nielsen J.F. , unpublished observations).

A dysfunction of the central motor pathways in diabetic patients could involve neurons at the cortical, corticospinal, or spinal level. Temporal summation of the paired stimuli is a likely explanation of the facilitation of the test CMAPs in control subjects. The possibility of temporal summation of corticocortical, corticospinal and spinal neurons depends on the membrane time constant. This constant expresses the falling phase of an action potential which is a purely passive membrane process. A long membrane time constant indicates a long action potential and consequently an increased probability for temporal summation. Bernander et al. [21] conducted a study where they took into account that neurons are embedded in a network of cells. They simulated the electrical behaviour of an anatomically reconstructed layer V cortical pyramidal cell receiving input from both excitatory and inhibitory cells firing spontaneously at 0-7 Hz. At the range of 7-80 ms depending on background activity the membrane time constant changed. Temporal summation on pyramidal cells could explain the facilitation at $30 \mathrm{~ms}$ observed in the present study.

At the segmental level descending volleys of Iwaves may produce temporal summation of the motorneuron. The appropriate length of time for temporal summation of excitatory postsynaptic potentials of the alpha motorneurons could be as short as 10 ms [22]. Therefore, it is unlikely that temporal summation of fast conducted I-waves generated by the conditioning stimulus and the test stimulus is the explanation of the facilitation at ISI of $30 \mathrm{~ms}$. However, there is some experimental evidence for the existence of slowly conducting corticospinal neurons which would make summation of the alpha motoneurons possible even after $30 \mathrm{~ms}$ [23].

Another explanation for the changes in the CMAPs is changes in excitability of the peripheral nerves [24]. In a normal peripheral nerve there are profound changes in the excitability during the first $100 \mathrm{~ms}$ after a submaximal electrical stimulation
[24-26]. Applying a stimulation intensity which evoked $25 \%$ of the maximal CMAP obtained from the APB, Potts et al. [25] found a period of hyperexcitability at ISIs of 5-20 ms and a period of hypoexcitability at ISIs of 20-100 ms with a maximum at an ISI of $50 \mathrm{~ms}$. The same temporal changes were found when $1 \%$ and $50 \%$ of the maximal CMAP were evoked. The paired transcranial magnetic stimulation protocol applied in this study results in two submaximal CMAPs. Although these two CMAPs are evoked by magnetic stimulation of the motor cortex and the stimulation intensities are of different size the transmission of the first impulse in the peripheral nerve probably alters the excitability of the nerve and thereby the size of the second (conditioned) CMAP. To our knowledge there are no studies of the changes in excitability of the peripheral nerves in diabetic patients, but the presence of peripheral neuropathy may change the excitability of peripheral motor nerves and thereby contribute to the loss of facilitation at ISIs of 30-50 ms. Further studies including paired magnetic and electrical stimulation of the peripheral nerves in diabetic patients are needed, in order to investigate the extent to which the excitability changes observed in the present study are caused by changes in the central or the peripheral part of the motor pathways.

At present the functional consequences of decreased excitability of the motor pathways are unknown, but they could contribute to motor disturbances such as decreased muscular endurance and slowing of movements in diabetic patients [27].

Acknowledgements. Mr. H. Kiilerich and Mr. B. Klemar are acknowledged for their excellent technical assistance, and the NOVO Nordisk Foundation and the Danish Diabetes Association for financial support for this study.

\section{References}

1. Dyck PJ, Kratz KM, Karnes JL et al. (1993) The prevalence by staged severity of various types of diabetic neuropathy, retinopathy, and nephropathy in a population-based cohort: the Rochester Diabetic Neuropathy Study. Neurology 43: 817-824

2. Murray NM (1992) The clinical usefulness of magnetic cortical stimulation. Electroencephalogr Clin Neurophysiol 85: 81-85 (Editorial)

3. Abbruzzese G, Schenone A, Scramuzza G et al. (1993) Impairment of central motor conduction in diabetic patients. Electroencephalogr Clin Neurophysiol 89: 335-340

4. Valls-Solé J, Pascual Leone A, Wassermann EM, Hallett M (1992) Human motor evoked responses to paired transcranial magnetic stimuli. Electroencephalogr Clin Neurophysiol 85 : $355-364$

5. Claus D, Weis M, Jahnke U, Plewe A, Brunholzl C (1992) Corticospinal conduction studied with magnetic double stimulation in the intact human. J Neurol Sci 111: 180-188

6. Dyck PJ, Sherman WR, Hallcher LM et al. (1980) Human diabetic endoneurial sorbitol, fructose, and myo-inositol related to sural nerve morphometry. Ann Neurol 8: 590-596 
7. Dyck PJ (1993) Quantitating severity of neuropathy. In: Dyck PJ, Thomas PK, Griffin JW (eds) Peripheral neuropathy, 3rd edn. Saunders, Philadelphia, pp 686-697

8. Miles DW, Mogensen CE, Gundersen HJG (1970) Radioimmunoassay for urinary albumin using a single antibody. Scand J Clin Lab Invest 26: 5-11

9. Stålberg E, Falck B (1993) Clinical motor nerve conduction studies. Methods in Clinical Neurophysiology 4: 61-80

10. Dyck PJ, O'Brien PC, Kosanke JL, Gillen DA, Karnes JL (1993) A 4, 2, and 1 stepping algorithm for quick and accurate estimation of cutaneous sensation threshold. Neurology 43: 1508-1512

11. Dyck PJ, Karnes JL, Daube J, O'Brien PC, Service FJ (1985) Clinical and neuropathological criteria for the diagnosis and staging of diabetic polyneuropathy. Brain 108: $861-880$

12. Dyck PJ (1988) Detection, characterization, and staging of polyneuropathy: assessed in diabetics. Muscle Nerve 11: 21-32

13. Nielsen JF (1994) Standardization of facilitation of compound muscle action potentials using a modified myometer during magnetic stimulation in healthy volunteers. Electroencephalogr Clin Neurophysiol 93: 75-79

14. Nakamura R, Noritake M, Hosoda Y, Kamakura, K, Nagata N, Shibasaki H (1992) Somatosensory conduction delay in central and peripheral nervous system of diabetic patients. Diabetes Care 15: 532-535

15. Virtaniemi J, Laakso M, Karja J, Nuutinen J, Karjalainen S (1993) Auditory brainstem latencies in type I (insulin-dependent) diabetic patients. Am J Otolaryngol 14: 413-418

16. Algan M, Ziegler O, Gehin P et al. (1989) Visual evoked potentials in diabetic patients. Diabetes Care 12: 227-229

17. Pozzessere G, Valle E, De Crignis S et al. (1991) Abnormalities of cognitive functions in IDDM revealed by $\mathrm{P} 300$ event-related potential analysis: comparison with short-la- tency evoked potentials and psychometric tests. Diabetes 40: $952-958$

18. Biessels GJ, Kappelle AC, Bravenboer B, Erkelens DW, Gispen WH (1994) Cerebral function in diabetes mellitus. Diabetologia 37: 643-650

19. Tchen PH, Fu CC, Chiu HC (1992) Motor-evoked potentials in diabetes mellitus. J Formos Med Assoc 91: 20-23

20. Pietravalle P, Morano S, Cristina G et al. (1993) Early complications in type 1 diabetes: central nervous system alterations preceded kidney abnormalities. Diabetes Res Clin Pract 21: 143-154

21. Bernander O, Douglas RJ, Martin KA, Koch C (1991) Synaptic background activity influences spatiotemporal integration in single pyramidal cells. Proc Natl Acad Sci USA 88: 11569-11573

22. Claus D, Weis M, Spitzer A (1991) Motor potentials evoked in tibialis anterior by single and paired cervical stimuli in man. Neurosci Lett 125: 198-200

23. Holmgren H, Larsson L, Pedersen S (1990) Late muscular responses to transcranial cortical stimulation in man. Electroencephalogr Clin Neurophysiol 75: 161-172

24. Morita H, Shindo M, Yanagawa S, Yanagisawa N (1993) Neuromuscular response in man to repetitive nerve stimulation. Muscle Nerve 16: 648-654

25. Potts F, Young RR, Shefner JM (1994) Long lasting excitability changes in human peripheral nerve. Muscle Nerve 17: 74-79

26. Stys PK, Ashby P (1990) An automated technique for measuring the recovery cycle of human nerves. Muscle Nerve 13: $750-758$

27. Mueller MJ, Minor SD, Sahrmann SA, Schaaf JA, Strube MJ (1994) Differences in the gait characteristics of patients with diabetes and peripheral neuropathy compared with age-matched controls. Phys Ther 74: 299-308 